\title{
Outlier Detection using Nonparametric Depth-Based Techniques in Hydrology
}

\author{
Insia Hussain $^{1}$ \\ College of Computer Science and Information System \\ Institute of Business Management, Karachi, Pakistan
}

\begin{abstract}
Several issues arise when extending the methods of outlier detection from a single dimension to a higher dimension. These issues include limited methods for visualization, marginal methods inadequacy, lacking a natural order and limitation in parametric modeling. The intension to overcome and address such limitations the nonparametric outlier identifier, based on depth functions, is introduced. These identifiers comprise of four threshold type outlyingness functions for outlier detection that are Mahalanobis distance, Tukey depth, spatial Mahalanobis depth, and projection depth. The object of the present research is the application of the proposed nonparametric technique in hydrology. The study is intended to be executed in two different frameworks that are multivariate hydrological data analysis and functional hydrological data analysis. The event of a flood is graphically represented by hydrograph whose components are used for computing flood characteristics that are peak $(p)$ and volume(v). These characteristics are frequently employed for the various types of analysis in the multivariate study. Whereas, hydrograph is exhaustively employed in the analysis of functional data so that all the important information regarding flood event are not missed while analysis. The proposed technique in a multivariate framework is applied to the bivariate flood characteristics $(p, v)$ while in functional framework proposed approach is applied to the initial two scores of principal components denoted as $\left(z_{1}, z_{2}\right)$, since initial two principal components capture major variation of data employed for analysis.
\end{abstract}

Keywords-Outlyingness functions; nonparametric techniques; flood characteristics; principal component scores; multivariate analysis; functional analysis

\section{INTRODUCTION}

The "outlier" observations in any data set is crucial to be detected and identified for nonparametric or parametric inferences. "Outliers" are the observations that are inconsistent or far from the majority of data points or within the chunk of data points with unusual behaviour. The presence of unusual observations in the data set acts as an outlier that can impact adversely the outcomes of estimation, inference, and testing procedures. Therefore, outliers are required to be identified and treated so that inferences are not violated due to unusual observations $[1,2]$.

Outliers identified marginally suffer inadequacy of checking, in each coordinate, an outlier can find to be nonoutlying. Approaches that are algorithmic and take into account underlying geometry are required. A suitable function of outlyingness may be formulated with a threshold specified. A suitable choice can be Mahalanobis distance which is a highly tractable function of outlyingness but constrained for having elliptical contours of symmetric outlyingness, even though whether the model under consideration is symmetric elliptically.

The author in [3] introduced a nonparametric technique which is based on functions of depth and orders the multidimensional data in center-outward. Higher depth represents higher centrality whereas lower depth greater outlyingness. One can associate with any depth function an equivalent function of outlyingness. For a suitable selection of depth function, actual geometrical structure and data shape are formed by equal outlyingness contours. In general, four different affine invariant functions of outlyingness were derived which are based on Mahalanobis distance outlyingness (MO), projection depth outlyingness (PO), halfspace or Tukey depth outlyingness (TO), and Spatial Mahalanobis outlyingness ( $\mathrm{SO}$ ). Related to these outlyingness functions the corresponding points are "outliers" having values of outlyingness exceed the constrained threshold of a particular function.

The nonparametric approaches introduced by [3] have been practiced by [4] and [5] in hydrology while [4] executed multivariate hydrological data analysis using two frequently employed flood characteristics; peak(p) \& volume(v), for the identification of unusual observations i.e. outliers.

The author in [5] came up with groundbreaking research and extended the work of [4] by conducting functional hydrological data analysis. The nonparametric outlier identification technique was practiced in hydrology by [5] in such a way that the initial two scores of principal components were employed for the detection of outliers in a functional context. In multivariate analysis, employed flood characteristics are dependent and mutually correlated whereas scores of principal components employed in functional analysis are uncorrelated.

The execution of research in the functional framework follows the claim made by [5] that the characteristic of flood use in conducting the multivariate hydrological study are computed by subjective approach and do not encounter the complete series of employed data set, therefore, inferences of multivariate study suffer lack of authenticity. Hence it is crucial to conduct research in a functional framework so that authentic estimation regarding the associated risk of flood is obtained by incorporating complete phenomena produced through employed data series. 
The objective carried by present research is the implementation of nonparametric techniques based on depth functions in both the context of a study that is a multivariate and functional framework using hydrological data of Kotri Barrage on Indus River in Pakistan.

\section{LITERATURE REVIEW}

The methods going to be presented are based mainly on the statistical notion of depth functions. These functions provide convenient ranking tools for ordering data variables. Depth functions were initiatively practiced in hydrology by [6]. Several techniques of univariate analysis were extended to execute multivariate analysis developed through analogy. The variables that are dependent mutually affect the performance badly when analysing data component-wise, whereas momentbased techniques required the moment's existence.

Review in detail regarding techniques use for conducting classical multivariate analysis, it is referred to follow $[7,8]$. Techniques that are developed on the basis of depth, avoid the earlier drawbacks science depth functions are ordered using multivariate inward and outward ranking [9]. Indeed, techniques based on depth aren't component-wise, also, they are affine invariant and moment-free. Numerous techniques of outlier detection are enabled by ranking based on depth. The number of depth function formulas have been derived for executing the multivariate study. Depth region location inference considered by [3] is evaluated on sample space. Description of connection and general treatment related to multivariate quantile and centre ranked functions can be studied through $[10,11]$. For other inferential applications of depth see $[12,13]$. Numerous studies conducted in hydrology using various nonparametric approaches. The functions based on depth have been recently employed for the detection of outliers by $[14,15]$. According to [16], nonparametric models are suitable for capturing subtle aspects related to the frequency estimation of a flood. Flood inundation and flood damage were analysed using hydrologically distributed models through nonparametric techniques [17]. Similar other studies recently conducted in hydrology for outlier detection and risk estimation using nonparametric approaches are $[18,19]$. Characteristics of drought evaluation were assessed in a multivariate context implementing a nonparametric approach by [20-22]. Further research of [23] discussed data cleaning of water consumption and estimation of uncertainty regarding hydrologic modeling. Depth notion in regression was practiced and the performance of runoff model was evaluated, see work of [24-26]. Author in [27] used parametric and nonparametric multivariate approaches for designing rainfall framework whereas [28] applied rank-based nonparametric techniques to study trends of rainfall.

Multidimensional data is reduced by of analysis of functional principal component (AFPC) techniques to attain an easy approach for analyzing hydrological data. Notable work includes profile classification of streamflow, minimum indicators selection and functional data analysis application on streamflow are the studies executed on the basis of AFPC. Simulation of drought interval and drought changes were analysed by [29,30]. [31-33] studied rainfall variability modeling, pattern identification, and outlier detection. Other relevant studies include work of [34-38], are also preferred for acquiring information about the useful application of AFPC in hydrology.

This paper is organized in such a way that the discussion regarding proposed methodologies is presented in Section 3. Section 4 provide description related to hydrological data employed for executing present research. Section 5 provides an application of the discussed methodology on employed hydrological data and obtained results are provided in Section 6 whereas Section 7 contain the conclusion drawn from the research.

\section{METHODOLOGY}

This section contains methods for computing bivariate series of flood characteristic $(p, v)$ and also bivariate series of principal component scores $\left(z_{1}, z_{2}\right)$. Both the computed series $(p, v)$ and $\left(z_{1}, z_{2}\right)$ are required for obtaining outliers in multivariate and functional context, respectively, using proposed threshold type nonparametric techniques which will also be discussed later in this section.

\section{A. Flood Characteristics}

The flood peak (p) and volume (v) are the fundamental and most studied flood characteristics [39-41] and their computation based on the work of [41].

The bivariate series $(p, v)$ are generated through hydrograph components using following formulas.

The flow peak series $p_{j}$ is calculated as.

$p_{j}=y_{h j}\left(t_{k}\right)$

where $y_{h j}\left(t_{k}\right)$ is the highest recorded observation of flow on a $k$ th day in a $j$ th year.

The flow volume series $v_{j}$ is calculated as.

$v_{j}=\sum_{l=S D_{j}}^{E D_{j}} y_{j}\left(t_{k}\right)-\frac{1}{2}\left(y_{i j}\left(t_{k}\right)+y_{f j}\left(t_{k}\right)\right)$

where $y_{j}\left(t_{k}\right)$ are the recorded observations of flow on a $k$ th day in a $j$ th year, $y_{s j}\left(t_{k}\right)$ and $y_{e j}\left(t_{k}\right)$ are the recorded observation of flow on starting $\left(S D_{j}\right)$ and ending day $\left(E D_{j}\right)$ respectively, in the $k$ th year of flood time span.

\section{B. Analysis of Functional Principal Component}

Analysis of principal component (APC) practices in a multivariate study for reducing the dimensionality through the computation of new variables which are the linear combination for original values so that the maximum of data variation could be captured. After the conversion of data as functions, analysis of functional principal component (AFPC) permits us to compute new functions so that special kind of variation for curve data could be revealed [5]. The AFPC method maximizes sample variance scores as orthonormal constraints. It divides the functional centred observations in orthogonal basis form and defined as follows.

Let functional observations be $y_{j}(t), j=1, \ldots, n$ obtained after smoothing the discrete observations $\left(y_{j}\left(t_{1}\right), \ldots, y_{j}\left(t_{T}\right)\right), j=1, \ldots, n$. By definition, the curve of mean is a same variation for most of the curves which can be 
fixed by centering. Let $\left(y_{j}^{*}(t)=y_{j}(t)-\bar{y}(t)\right)_{j=1, \ldots, n}$ be functional centered observations where $\bar{y}(t)$ represents the function of mean for $\left(y_{1}(t), \ldots, y_{n}(t)\right)$. Now AFPC is applied to $\left(y_{j}^{*}(t)\right)_{j=1, \ldots, n}$ for creating a set of small functions, known as harmonics which reveals the type of variation important for analysis. The first principal component $\left(y_{j}^{*}(t)\right)_{j=1, \ldots, n}$ denoted as $w_{1}(t)$ be a function so that variance regarding corresponding scores $z_{j, 1}$ of real value is as follows.

$z_{j, 1}=\int_{C} w_{1}(s) y_{j}^{*}(s) d s, j=1, \ldots, n$

is maximized under $\int_{C} w_{1}(s)^{2} d s=1$ constraint. The next $w_{l}(t)$; a principal component computed by maximization of variance related to corresponding scores $z_{j, l}$ :

$z_{j, l}=\int_{C} w_{l}(s) y_{j}^{*}(s) d s, j=1, \ldots, n$

under $\int_{C} w_{l}(s) w_{k}(s) d s=0, l \geq 2, l \neq k$ constraints.

\section{Detection of Outliers}

The approaches for detection of outliers employed by [4] in the multivariate context was adapted by [5] in functional context; applying functions of outlyingness on the scores of initial two principal components. The purpose of this adaption is to create a comparison between multivariate and functional results.

Functions of outlyingness in a multivariate context were described and employed for detecting outliers. These functions have values ranging $[0,1]$ interval. The outlyingness of a particular point is measured related to the whole sample. A value of outlyingness close to 1 shows high outlyingness, and a value close to 0 shows centrality. An observation is determined to be an outlier by defining a threshold i.e. the outlyingness value corresponds to an outlier must exceed their respective threshold values. Reference [3] introduced outlyingness functions which are based on the functions of depth, are going to be presented in the following section.

1) Outlyingness functions: A depth function is transformed to depth outlyingness for a $\mathrm{F}$ given distribution and $x \in R^{d}$. Reference [3] studied as follows.

a) Half space

$O_{\text {HO }}(x, F)=1-2 \mathrm{HO}(x, F)$

b) Mahalanobis

$O_{M O}(x, F)=d_{A(F)}^{2}(x, \mu(F)) /\left[1+d_{A(F)}^{2}(x, \mu(F))\right]$

c) Projection

$O_{P O}(x, F)=P O(x, F) /[1+P O(x, F)]$

where $H O(., F), d^{2}{ }_{A(F)}(., \mu(F))$ and $P O(., F)$ are given by [4], a location measure is $\mu(F)$ and $A(F)$ is non-singular measure of scale matrix.

Spatial

$O_{s o}(x, F)=\|E(\operatorname{Sign}(x-X))\|$

d) Spatial Mahalanobis
$O_{M S}(x, F)=\| E\left[\operatorname{Sign}\left(\mathbf{C}^{-\frac{1}{2}}(x-X)\right] \|\right.$

where the Euclidean norm is $\|\|,$.$F -distribution is X$ and the sign multidimensional function is $\operatorname{Sign}($.$) given by$ $\operatorname{Sign}(x)=x /\|x\|$ if $x \neq 0$ and $\operatorname{Sign}(0)=0$ also, $\mathbf{C}$ is any positive definite affine invariant $d \times d$ symmetric matrix.

2) Threshold: An essential step in the detection of an outlier is the appropriate selection of the threshold. It relates to true positive and false positive rates. $\alpha_{n}$ denoted for a false positive arbitrary rate which is defined as the proportion of misidentified nonoutliers as outliers. This constant relates closely to the $\varepsilon_{n}$ true positive rate by which the theoretical proportion for real outliers are represented (also known as contaminants). Ideally, $\alpha_{n}$ suppose to be smaller than $\varepsilon_{n}$. Reference [3] fixed the false outliers' ratio $\delta=\alpha_{n} / \varepsilon_{n}$ and also used another coefficient $\beta=\varepsilon_{n} \sqrt{n}$, in order to define a threshold for the values of outlyingness as $\left(1-\alpha_{n}\right)$ quantile.

$\rho_{n}=F_{O(X, F)}^{-1}\left(1-\alpha_{n}\right)=F_{O(X, F)}^{-1}\left(1-\delta \beta_{n} / \sqrt{n}\right)$

where false positive rate $\alpha_{n}$ is represented as $\alpha_{n}=$ $\delta \beta_{n} / \sqrt{n}$ and true positive rate $\varepsilon_{n}$ represented as $\varepsilon_{n}=n \varepsilon_{n} / n$; a number of true outliers are $n \varepsilon_{n}$ and a number of observations are $n$, in such a way that $\alpha_{n}<\varepsilon_{n}$. For further calculations and applications, readers are referred to follow [4].

\section{DATA DESCRIPTION}

The major source of hydrological data is daily streamflow. The daily flow data series of the Kotri barrage are available from Sindh Irrigation department, Sindh Secretariat, Karachi, Pakistan.

A daily flow observations $\left(\mathrm{m}^{3} \mathrm{~s}^{-1}\right)$ of Kotri barrage which is located between Jamshoro and Hyderabad in Sindh province on the Indus River, Pakistan. It has a discharge capacity of 875,000 cusecs (i.e. approximately $24800 \mathrm{~m}^{3} \mathrm{~s}^{-1}$ ). Fig. 1 indicates the geographical location of the Kotri Barrage.

Some studies contain data of complete year while some consider section of a year having high flow observations. Hydrological data observations of the present study contain a duration of 6 months (i.e.T $=183$ days) per year spanning 1977 to 2017 (i.e. $n=41$ years) since high flow period is observed during the months April to September, in Pakistan. The series of observations are $Y_{j}=\left(y_{j}\left(t_{1}\right), \ldots, y_{j}\left(t_{T}\right)\right)$, $j=1, \ldots, n, k=1, \ldots, T$, where $n=41$ years, $T=183$ days and $y_{j}\left(t_{k}\right)$ is the recorded flow observation on $t_{k}$ day in the $j$ th year. Before any computation is performed the streamflow observations which are recorded on measurement scale in cusec (a volume flow rate) are required to be converted into cubic meter per second $\left(\mathrm{m}^{3} \mathrm{~s}^{-1}\right)$. 


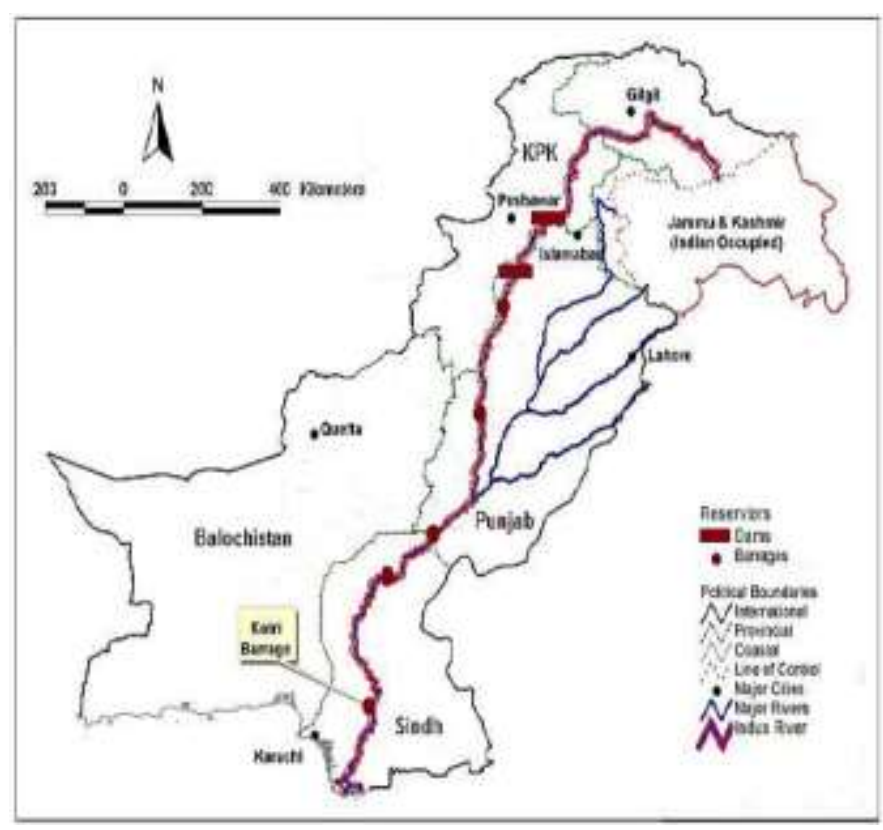

Fig. 1. Geographical Location of Kotri Barrage.

\section{APPLICATION}

The two most studied and examined characteristics of the flood that is peak (p) and volume (v) are focused here. The series of bivariate (p,v) are computed by using (1) and (2) and results are displayed in Table I.

According to [4], an approach developed by [3] are based on the function of depth outlyingness and the threshold corresponded. The four functions of depth outlyingness are evaluated for the $(\mathrm{p}, \mathrm{v})$ series of bivariate observation i.e., Mahalanobis (MO), Projection (PO), Spatial (SO) and Tukey (TO). The values of depth outlyingness correspond to each $(p, v)$ observation for years 1977-2017 are reported in the last four columns of Table I. The thresholds correspond to each outlyingness functions are computed by selecting $15 \%$ false outlier ratio and the number of true outliers as 5 , this selection is similar to the choices made by [4] in such a way that the outlyingness value corresponds to an outlier must exceed their respective threshold values.

Hence, $98 \%$ quantile is a corresponding threshold for the values of outlyingness. The computed values of the threshold for MO, PO, SO \& TO are 0.9412, 0.9040, 0.9719, and 0.9444 , respectively. The values of threshold approximately remain constant if the number of true outliers is considered greater than 5 with changed false outlier ratio i.e. $5 \%, 10 \%$ and $20 \%$. The detected outliers correspond to MO, PO, SO \& TO with respect to their respective threshold values are graphically displayed by Fig. 2 .

Reference [5] employed the procedure for detecting outliers which are based on the function of depth outlyingness and the threshold corresponded. As discussed earlier and also practiced in preceding section, four functions of depth outlyingness are evaluated for the series of the bivariate score $\left(z_{1}, z_{2}\right)$ i.e., Mahalanobis (MO), Projection (PO), Spatial (SO) and Tukey (TO).
TABle I. Multivariate Results For Flood PeaK and Volume

\begin{tabular}{|c|c|c|c|c|c|c|}
\hline Year & Peak & Volume & MO & PO & SO & TO \\
\hline 1977 & 7490 & 248765 & 0.0979 & 0.5424 & 0.4134 & 0.4634 \\
\hline 1978 & 15747 & 249063 & 0.8782 & 0.8631 & 0.4183 & 0.9512 \\
\hline 1979 & 7342 & 305373 & 0.4843 & 0.7099 & 0.6352 & 0.7561 \\
\hline 1980 & 5776 & 170479 & 0.0852 & 0.2978 & 0.0255 & 0.2195 \\
\hline 1981 & 7149 & 246426 & 0.1473 & 0.5673 & 0.3586 & 0.5610 \\
\hline 1982 & 5560 & 129340 & 0.1783 & 0.4059 & 0.2671 & 0.3171 \\
\hline 1983 & 9367 & 260061 & 0.1161 & 0.5753 & 0.4844 & 0.5610 \\
\hline 1984 & 7913 & 290839 & 0.2922 & 0.6491 & 0.5849 & 0.7073 \\
\hline 1985 & 3662 & 126804 & 0.3419 & 0.5121 & 0.3348 & 0.5610 \\
\hline 1986 & 10160 & 185277 & 0.6149 & 0.7608 & 0.1526 & 0.9024 \\
\hline 1987 & 2771 & 128432 & 0.4982 & 0.6217 & 0.2893 & 0.9024 \\
\hline 1988 & 14527 & 467773 & 0.6348 & 0.7848 & 0.7808 & 0.8049 \\
\hline 1989 & 6276 & 112997 & 0.3567 & 0.6141 & 0.3900 & 0.6585 \\
\hline 1990 & 6355 & 243994 & 0.3066 & 0.6250 & 0.3110 & 0.6585 \\
\hline 1991 & 5309 & 276870 & 0.6496 & 0.7430 & 0.5363 & 0.9512 \\
\hline 1992 & 15241 & 618581 & 0.8350 & 0.8484 & 0.8783 & 0.9024 \\
\hline 1993 & 9617 & 217016 & 0.3713 & 0.6765 & 0.1981 & 0.7073 \\
\hline 1994 & 19109 & 921882 & 0.9482 & 0.9043 & 0.9756 & 0.9512 \\
\hline 1995 & 17998 & 483519 & 0.7882 & 0.8274 & 0.8288 & 0.8537 \\
\hline 1996 & 8520 & 417460 & 0.7610 & 0.8073 & 0.7321 & 0.9024 \\
\hline 1997 & 6898 & 145428 & 0.2501 & 0.5854 & 0.1765 & 0.4634 \\
\hline 1998 & 6263 & 181396 & 0.0444 & 0.2874 & 0.1065 & 0.2195 \\
\hline 1999 & 4133 & 59546 & 0.4171 & 0.5835 & 0.5856 & 0.8049 \\
\hline 2000 & 1372 & 27595 & 0.5406 & 0.6543 & 0.8807 & 0.9512 \\
\hline 2001 & 1969 & 39701 & 0.4927 & 0.6301 & 0.6815 & 0.8537 \\
\hline 2002 & 2581 & 32254 & 0.4782 & 0.6272 & 0.7895 & 0.8537 \\
\hline 2003 & 4171 & 146269 & 0.3006 & 0.4783 & 0.1627 & 0.5122 \\
\hline 2004 & 898 & 30884 & 0.5784 & 0.6626 & 0.8236 & 0.9512 \\
\hline 2005 & 6800 & 236405 & 0.1577 & 0.5614 & 0.2491 & 0.5122 \\
\hline 2006 & 7922 & 154970 & 0.3857 & 0.6698 & 0.0733 & 0.7073 \\
\hline 2007 & 3323 & 147582 & 0.4653 & 0.5966 & 0.1364 & 0.8049 \\
\hline 2008 & 2882 & 87966 & 0.4016 & 0.5513 & 0.4880 & 0.6098 \\
\hline 2009 & 2111 & 36592 & 0.4870 & 0.6291 & 0.7316 & 0.8049 \\
\hline 2010 & 28244 & 694249 & 0.9404 & 0.9044 & 0.9267 & 0.9512 \\
\hline 2011 & 4459 & 45005 & 0.5054 & 0.6305 & 0.6391 & 0.9512 \\
\hline 2012 & 2115 & 22688 & 0.5078 & 0.6420 & 0.9725 & 0.9512 \\
\hline 2013 & 8475 & 174738 & 0.3751 & 0.6731 & 0.0634 & 0.6585 \\
\hline 2014 & 3005 & 24519 & 0.5024 & 0.6425 & 0.9248 & 0.9512 \\
\hline 2015 & 14155 & 325111 & 0.6981 & 0.7957 & 0.6819 & 0.8537 \\
\hline 2016 & 3257 & 86015 & 0.3600 & 0.5389 & 0.5355 & 0.5610 \\
\hline 2017 & 5730 & 97637 & 0.3715 & 0.5963 & 0.4407 & 0.7561 \\
\hline
\end{tabular}

Legend

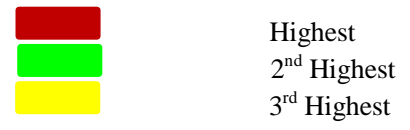




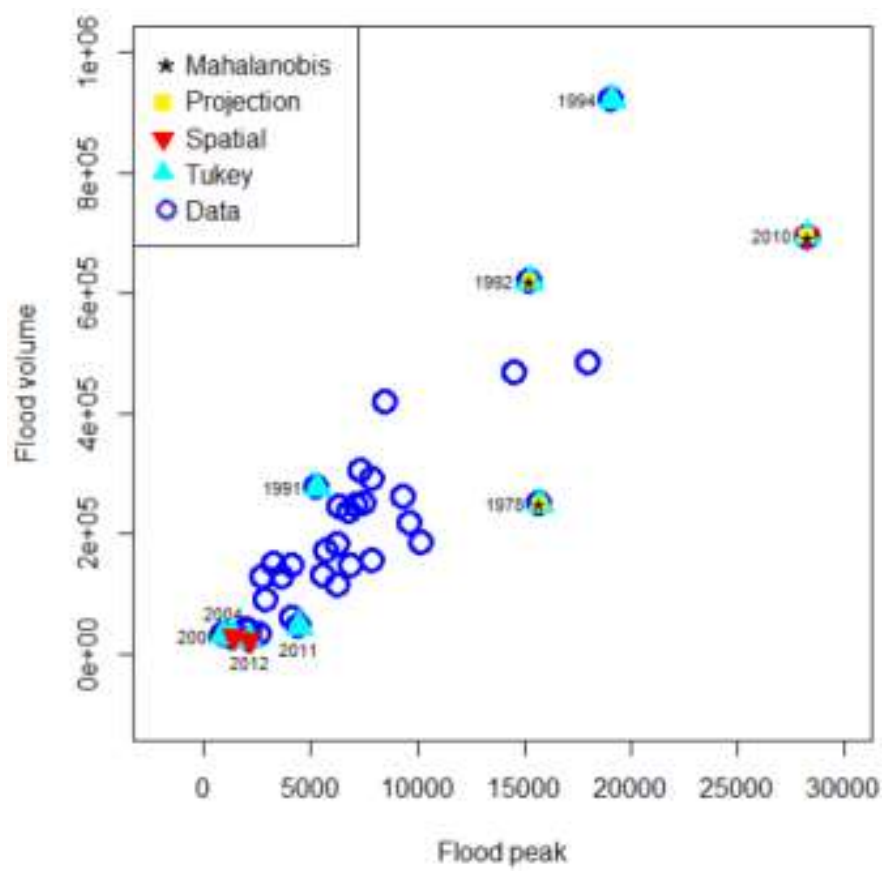

Fig. 2. Detected Outliers using Flood Peak and Volume.

The thresholds correspond to each outlyingness functions are computed by selecting $15 \%$ false outlier ratio and the number of true outliers as 5 , this selection is similar to the choices made by [4] in such a way that the outlyingness value corresponds to an outlier must exceed their respective threshold values. Hence, $98 \%$ quantile is a corresponding threshold for the values of outlyingness. The computed values of the threshold for MO, PO, SO \& TO are 0.9106, 0.8905, 0.9264 , and 0.9444 , respectively. The values of threshold approximately remain constant if the number of true outliers is considered greater than 5 with changed false outlier ratio i.e. $5 \%, 10 \%$ and $20 \%$. The computed outlyingness values of MO, PO, SO \& TO for years 1977-2017 are tabulated in Table II whereas Fig. 3 displays the detected outliers correspond to $\mathrm{MO}, \mathrm{PO}, \mathrm{SO} \& \mathrm{TO}$ with respect to their respective threshold values.

\section{RESULTS}

\section{A. Multivariate Result}

The year 1994 contain outlyingness values greater than their respective threshold values by MO, PO \& SO functions. Several years including years 1978, 1994, 2010 and 2012 are detected by TO function as outliers. The year 2010 is detected by $\mathrm{MO}$ and PO, and year 2012 is detected by SO functions as the closest value of outlyingness with respect to their threshold values. In addition, the year 1978 corresponds to the third highest MO and PO values whereas the year 2010 correspond the third highest SO value compare to their respective threshold values. Hence, it can objectively be inferred from Table I that the years 1994 and 2010 are identified as outliers by all the four functions of outlyingness. Whereas, the year 1978 is detected by the three and the year 2012 is detected by the two functions of outlyingness. For illustrative purpose a scatter plot constructed between bivariate (p,v) series (i.e. flood peak and flood volume) is displayed through Fig. 2 so that the above interpretation can explicitly comprehensible. The years 1978, 1990, 1994, 2000, 2004, 2010, 2011, 2012 and 2014 computed as outliers by the outlyingness functions, among them the years 1978 and 1992 are present outside compare to the rest of the years whereas the years 1994 and 2010 are appear as outliers.

TABLE II. FUnCtIONAL RESUlts For PRINCIPAL COMPONENT $\left(z_{1}, z_{2}\right)$

\begin{tabular}{|c|c|c|c|c|c|c|}
\hline Year & $z_{1}$ & $z_{2}$ & MO & PO & SO & TO \\
\hline 1977 & -2.29 & -2.339 & 0.1671 & 0.5215 & 0.3226 & 0.5122 \\
\hline 1978 & 13.09 & -10.776 & 0.8342 & 0.8565 & 0.8512 & 0.9024 \\
\hline 1979 & 6.218 & 7.085 & 0.6323 & 0.8256 & 0.6295 & 0.7561 \\
\hline 1980 & -3.056 & 0.173 & 0.1077 & 0.2189 & 0.0381 & 0.1707 \\
\hline 1981 & 9.388 & 8.516 & 0.7435 & 0.8548 & 0.7702 & 0.9512 \\
\hline 1982 & -5.564 & 3.525 & 0.4119 & 0.5763 & 0.4923 & 0.7073 \\
\hline 1983 & 7.676 & -2.302 & 0.4697 & 0.7249 & 0.6046 & 0.6585 \\
\hline 1984 & -3.352 & -4.623 & 0.3994 & 0.6705 & 0.5732 & 0.8537 \\
\hline 1985 & -9.07 & -1.06 & 0.5201 & 0.5805 & 0.7348 & 0.9512 \\
\hline 1986 & -3.226 & -2.818 & 0.2465 & 0.5537 & 0.3994 & 0.6585 \\
\hline 1987 & 1.832 & 5.992 & 0.4786 & 0.7791 & 0.5310 & 0.6585 \\
\hline 1988 & 7.617 & -3.672 & 0.5177 & 0.7498 & 0.6238 & 0.7073 \\
\hline 1989 & -5.09 & 0.182 & 0.2501 & 0.3459 & 0.2150 & 0.3171 \\
\hline 1990 & 6.42 & -1.683 & 0.3743 & 0.6943 & 0.5049 & 0.5610 \\
\hline 1991 & 20.652 & 9.365 & 0.8839 & 0.8928 & 0.9015 & 0.9512 \\
\hline 1992 & 28.743 & 3.228 & 0.9157 & 0.8888 & 0.9422 & 0.9512 \\
\hline 1993 & 7.174 & 7.721 & 0.6788 & 0.8378 & 0.6965 & 0.8049 \\
\hline 1994 & 7.345 & -21.331 & 0.9217 & 0.8938 & 0.9077 & 0.9512 \\
\hline 1995 & 9.233 & -6.894 & 0.6926 & 0.8068 & 0.7436 & 0.8049 \\
\hline 1996 & 7.365 & -4.316 & 0.5350 & 0.7577 & 0.6280 & 0.7561 \\
\hline 1997 & -4.721 & -0.359 & 0.2244 & 0.2994 & 0.2017 & 0.3659 \\
\hline 1998 & 9.949 & 8.385 & 0.7490 & 0.8559 & 0.7998 & 0.9024 \\
\hline 1999 & -6.452 & 1.793 & 0.3800 & 0.4861 & 0.4139 & 0.5610 \\
\hline 2000 & -10.234 & 2.782 & 0.6053 & 0.6467 & 0.8525 & 0.9512 \\
\hline 2001 & -5.921 & 6.952 & 0.6195 & 0.7290 & 0.7311 & 0.9512 \\
\hline 2002 & -9.377 & 1.845 & 0.5479 & 0.5970 & 0.7410 & 0.8537 \\
\hline 2003 & -3.783 & -0.194 & 0.1559 & 0.2193 & 0.0807 & 0.2195 \\
\hline 2004 & -10.197 & 2.608 & 0.6002 & 0.6415 & 0.8266 & 0.9512 \\
\hline 2005 & 0.865 & 2.131 & 0.1073 & 0.6408 & 0.3050 & 0.4634 \\
\hline 2006 & -6.466 & -2.704 & 0.4169 & 0.6282 & 0.6128 & 0.8537 \\
\hline 2007 & 0.884 & 6.854 & 0.5359 & 0.7897 & 0.5904 & 0.9024 \\
\hline 2008 & -8.884 & 0.856 & 0.5077 & 0.5714 & 0.6634 & 0.7561 \\
\hline 2009 & -8.824 & 1.963 & 0.5224 & 0.5829 & 0.6608 & 0.8049 \\
\hline 2010 & -0.407 & -19.296 & 0.9007 & 0.8898 & 0.8743 & 0.9512 \\
\hline 2011 & -6.425 & -1.158 & 0.3601 & 0.4990 & 0.4612 & 0.6098 \\
\hline 2012 & -9.277 & -0.121 & 0.5251 & 0.5829 & 0.7346 & 0.9024 \\
\hline 2013 & -6.19 & -2.373 & 0.3862 & 0.5996 & 0.5283 & 0.7561 \\
\hline 2014 & -7.242 & 1.574 & 0.4233 & 0.5073 & 0.4995 & 0.6098 \\
\hline 2015 & 1.404 & -1.802 & 0.0946 & 0.5918 & 0.3483 & 0.4634 \\
\hline 2016 & -3.317 & 5.357 & 0.4567 & 0.7064 & 0.5341 & 0.9024 \\
\hline 2017 & -6.487 & 0.934 & 0.3596 & 0.4502 & 0.3736 & 0.5610 \\
\hline
\end{tabular}

Legend

\begin{tabular}{|l}
\hline Highest \\
\hline $2^{\text {nd }}$ Highest \\
\\
$3^{\text {rd }}$ Highest
\end{tabular}




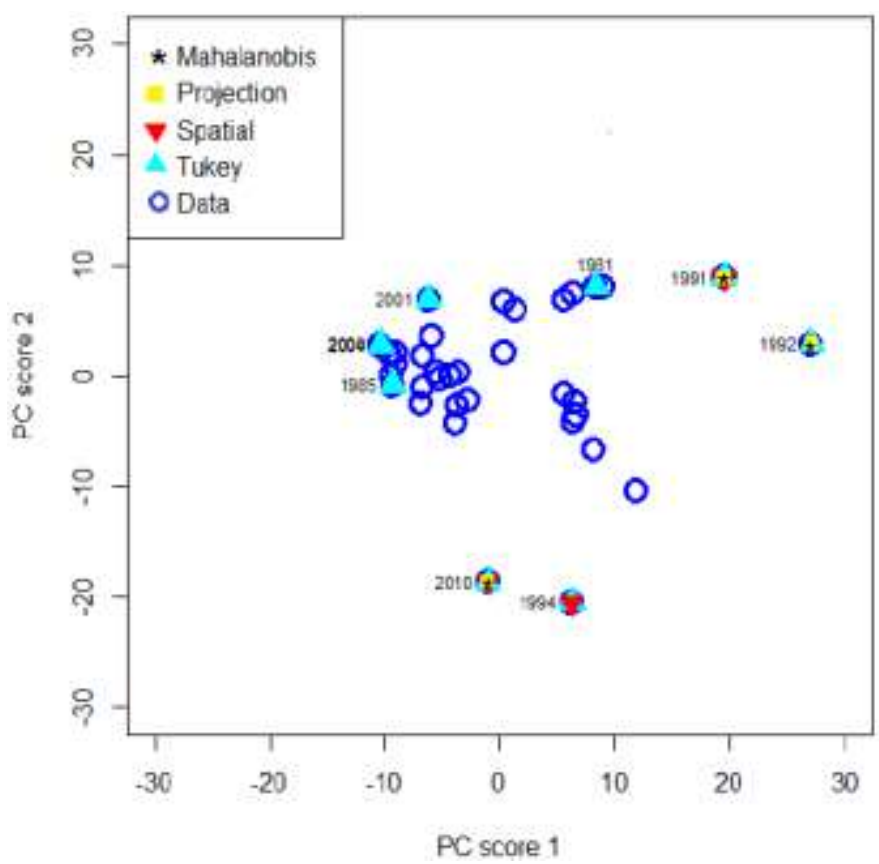

Fig. 3. Detected Outliers using Principal Component Scores.

\section{B. Functional Result}

It is observed that the year 1994 contain outlyingness values greater than their respective threshold values by $\mathrm{MO}$ and PO functions whereas outlyingness value of the year 1992 is greater than the threshold value by SO function. Several years including 1991, 1992, 1994 and 2010 are detected by TO function as outliers. The year 1991 is detected by the PO, the year 1992 is detected by MO and the year 1994 is detected by the SO functions as a second highest outlyingness values compare to their respective threshold values. In addition, the year 2010 corresponds to the third highest MO and PO values, whereas the year 1991 corresponds to the third highest SO outlyingness value according to their respective threshold values.

Hence, it can distinctly be inferred from the values of Table II, the year 1994 is detected by all the four outlyingness functions as an outlier. Whereas the years 1991, 1992 and 2010 are identified as outliers by the three outlyingness functions. Above interpretation can better be comprehended by the scatter plot constructed between scores of initial two principal components (i.e. PC score $1 \&$ score 2) and represented by Fig. 3 which reveals that the years 1981, 1985, 1991, 1992, 1994, 2000, 2001, 2004 and 2010 computed as outliers by the outlyingness functions, among them the years 1991 and 1992 are present outside compare to the rest of the years whereas the years 1994 and 2010 are appear as outliers.

The functional results are almost consistent with the results of the multivariate framework such that the years 1992, 1994 and 2010 have been detected as the most unusual flows in both the multivariate and functional context.

\section{CONCLUSION}

The nonparametric techniques based on depth function for outlier identifiers have been practiced in two different frameworks of study that are multivariate hydrological data analysis and functional hydrological data analysis. The identification of outlier is essential for the appropriate selection of suitable hydrologic models so that risk associated with flood events can be authentically estimated. The methods employed in the present research are multivariate methods that are superior to previously practiced classical methods that were moment-based, follow normality assumption and component-wise techniques. The implemented techniques are based on depth function notion, free of moment, do not require normality assumption, and also affine invariant.

The proposed approaches have been implemented in two different frameworks of analysis. The intention of executing this study is to gauge the performance of proposed methodologies in both multivariate and functional context. The two most widely practice flood characteristics in hydrological analysis, peak (p) \& volume (v) have been included to execute study in multivariate hydrological data analysis. Besides this, two initial scores of principal components $\left(z_{1}, z_{2}\right)$ used as a series of bivariate variables for executing functional hydrological data analysis since initial two principal components have a capability to capture major variation of data employed for analysis.

The outliers of both the framework are almost consistent but the results of functional analysis can be considered more reliable since it is based on complete information of flood hydrograph whereas flood characteristics $(p, v)$ are not able to generate hydrograph even though more than two characteristics of flood are included in study. Nevertheless, the multivariate results cannot be ignored and must be employed in a parallel complement to functional results so that dynamics of a hydrological event can be analysed to attain comprehensive information related to causes of flood.

REFERENCES

[1] V. Barnett, and T. Lewis, Outliers in Statistical Data, $3^{\text {rd }}$ ed., John Wiely, Chichester, U.K, 1998.

[2] V. Barnett, Environmental Statistics: Methods and Applications, John Wiely, Chichester, U.K, 2004.

[3] X. Dang, and R. Serfling, "Nonparametric depth-based multivariate outlier identifiers, and masking robustness properties," Journal of Statistical Planning and Inference, vol. 140, no. 1, pp. 198-213, 2010. doi: 10.1016/j.jspi.2009.07.004.

[4] F. Chebana and T. B. Ouarda, "Depth-based multivariate descriptive statistics with hydrological applications," Journal of Geophysical Research, vol. 116, D10120, 2011b. doi:10.1029/2010JD015338.

[5] F. Chebana, S. Dabo-Niang and T. B. Ouarda, "Exploratory functional flood frequency analysis and outlier detection," Water Resources Research, vol. 48, no. 4, W04514, 2012. doi:10.1029/2011WR011040.

[6] F. Chebana, and T. B. Ouarda, "Depth and homogeneity in regional flood frequency analysis," Water Resources Research, vol. 44, W11422, 2008. doi:10.1029/2007WR006771.

[7] T. W. Anderson, An Introduction to Multivariate Statistical Analysis, 2nd ed., John Wiley, Chichester, U. K, 1984.

[8] M. J. Schervish, "A review of multivariate analysis," Statistical Science, vol. 2, no. 4, pp. 413-417, 1987. doi:10.1214/ss/1177013111.

[9] Y. Zuo, and R. Serfling, "General notions of statistical depth function," Annals of Statistics, vol. 28, no. 2, pp. 461-482, $2000 \mathrm{~b}$. doi:10.1214/aos/1016218226.

[10] R.Y. Liu, J. M. Parelius, and K. Singh, "Multivariate analysis by data depth: Descriptive statistics, graphics and inference," Annals of Statistics, vol. 27, no. 3, pp. 783-858, 1999. 
[11] J. Zhang, "Some extensions of Tukey's depth function," Journal of Multivariate Analysis vol. 82, no. 1, pp. 134-165, 2002.

[12] Y. Zuo, and R. Serfling, "On the performance of some robust nonparametric location measures relative to a general notion of multivariate symmetry," Journal of Statistical Planning and Inference, vol. 84, no. 1-2, pp. 55-79, 2000a. doi:10.1016/S03783758(99)00142-1.

[13] C. H, Müller, "Depth estimators and tests based on the likelihood principle with application to regression," Journal of Multivariate Analysis, vol. 95, no. 1, pp. 153-181, 2005.

[14] I. Hussain, and M. Uddin, "Functional and multivariate hydrological data visualization and outlier detection of Sukkur Barrage," International Journal of Computer Applications, vol. 178, no. 28, pp. 20-29, 2019. doi:10.5120/ijca2019919097.

[15] I. Hussain, "Outlier detection using graphical and nongraphical functional methods in hydrology," International Journal of Advanced Computer Science and Applications, vol. 10, no. 12, pp. 438-445, 2019. doi: 10.14569/IJACSA.2019.0101259.

[16] G. A. Griffiths, S. K. Singh, and A. I. McKerchar, "Flood frequency estimation in New Zealand using a region of influence approach and statistical depth functions," Journal of Hydrology, vol. 589, pp. 125-187, 2020. doi 10.1016/j.jhydrol.2020.125187.

[17] M. Karamouz, F.ASCE, F. Ahmadvand, and Z. Zahmatkesh, "Distributed hydrologic modelling of coastal flood inundation and damage: Nonstationary approach," Journal of Irrigation and Drainage Engineering, vol. 143, no. 8, 2017. doi: 10.1061/(ASCE)IR.19434774.0001173.

[18] W. Fan, L. Heng, D. Chao \& D. Lieyun,"Knowledge representation using non-parametric Bayesian networks for tunneling risk analysis," Reliability Engineering and System Safety, Elsevier, vol. 191(C), 2019. doi: 10.1016/j.ress.2019.106529.

[19] L. Millán-Roures, I. Epifanio, and V. Martínez, "Detection of Anomalies in Water Networks by Functional Data Analysis," Mathematical Problems in Engineering, 2018. doi: org/10.1155/2018/5129735.

[20] Y. Zhang, S. Huang, Q. Huang, G. Leng, H. Wang, and L. Wang, "Assessment of drought evolution characteristics based on a nonparametric and trivariate integrated drought index," Journal of Hydrology, vol. 579, 2019. doi 10.1016/j.jhydrol.2019.124230.

[21] K. T. Peterson, V. Sagan, and J. J. Sloan, "Deep learning-based water quality estimation and anomaly detection using Landsat-8/Sentinel-2 virtual constellation and cloud computing," GIScience \& Remote Sensing, vol. 57, no. 4, pp. 510-525, 2020 . doi: 10.1080/15481603.2020.1738061.

[22] J. Rhee, K. Park, S. Lee, S. Jang, and S. Yoon, "Detecting hydrological droughts in ungauged areas from remotely sensed hydro-meteorological variables using rule-based models," Natural Hazards, vol. 57, 2020. doi:10.1007/s11069-020-04114-5.

[23] R. Padulano, G. D. Giudice, "A nonparametric framework for water consumption data cleansing: an application to a smart water network in Naples (Italy)," Journal of Hydroinformatics, vol. 22, no. 4, pp. 666680, 2020. doi: org/10.2166/hydro.2020.133.

[24] S. Samadi, D. L. Tufford, and G. J. Carbone, "Estimating hydrologic model uncertainty in the presence of complex residual error structures," Stochastic Environmental Research and Risk Assessment, vol. 32, pp. 1259-1281, 2018. doi: org/10.1007/s00477-017-1489-6.

[25] Y. Zuo, "On general notions of depth for regression," arXiv e-prints, 2018.

[26] S. Pool, M. Vis, and J. Seibert, "Evaluating model performance: towards a non-parametric variant of the Kling-Gupta efficiency," Hydrological
Sciences Journal, vol. 63, no. 13-14, pp. 1941-1953, 2018. doi: $10.1080 / 02626667.2018 .1552002$.

[27] M. A. Sherly, S. Karmakar, T. Chan, and C. Rau, "Design Rainfall Framework Using Multivariate Parametric-Nonparametric Approach," Journal of Hydrologic Engineering, vol. 21, no. 1, 2016. doi: org/10.1061/(ASCE)HE.1943-5584.0001256.

[28] W.W.U.I. Wickramaarachchi, T.U.S. Peiris, and S. Samita, "Rainfall Trends in the North-Western and Eastern Coastal Lines of Sri Lanka Using Non - Parametric Analysis," Tropical Agricultural Research, vol. 31, no. 2, pp. 41-54, 2020. doi: 10.4038/tar.v31i2.8366.

[29] U. Beyaztas, and Z. M. Yaseen, "Drought interval simulation using functional data analysis" Journal of Hydrology, vol. 579, 2019. doi: 10.1016/j.jhydrol.2019.124141.

[30] J. Xia, P. Yang, C. Zhan and Y. Qiao, "Analysis of changes in drought and terrestrial water storage in the Tarim River Basin based on principal component analysis," Hydrology Research, vol. 50 no. 2, pp. 761-777, 2019. doi: 10.2166/nh.2019.033.

[31] M. A. Hael, "Modeling of rainfall variability using functional principal component method: a case study of Taiz region, Yemen," Modeling Earth Systems and Environment, vol. 2, no. 7, 2020, doi: 10.1007/s40808-020-00876-w.

[32] M. A. Hael1, Y. Yongsheng, and B. I. Saleh, "Visualization of rainfall data using functional data analysis", SN Applied Sciences, vol. 2, no. 2, 2020. doi:10.1007/s42452-020-2238-x.

[33] S.M. Shaharudin, N. Ahmad, N.H. Zainuddin, and N.S. Mohamed, "Identification of rainfall patterns on hydrological simulation using robust principal component analysis", Indonesian Journal of Electrical Engineering and Computer Science, vol. 11, no. 3, pp.1162-1167, 2018. doi: 10.11591/ijeecs.v11.i3.pp1162-1167.

[34] J. Suhaila, Application of Functional Data Analysis in Streamflow Hydrograph. In: Kor LK., Ahmad AR., Idrus Z., Mansor K. (eds) Proceedings of the Third International Conference on Computing, Mathematics and Statistics (iCMS2017). (2019) Springer, Singapore.

[35] M. A. T. M. T. Rahman, S. Hoque, and A. H. M. Saadat, "Selection of minimum indicators of hydrologic alteration of the Gorai river, Bangladesh using principal component analysis," Sustainable Water Resources Management, 2017. doi: org/10.1007/s40899-017-0079-6.

[36] S. Xiao, Z. Lu, and L. Xu, "Multivariate sensitivity analysis based on the direction of eigen space through principal component analysis," Reliability Engineering \& System Safety, vol. 165, 2017. doi: 10.1016/j.ress.2017.03.011.

[37] S. K. Sharma, S. Tignath, S. Gajbhiye, and R. Patil, "Application of principal component analysis in grouping geomorphic parameters of Uttela watershed for hydrological modeling," International Journal of Remote Sensing \& Geoscience, vol. 2, no. 6, 2013.

[38] C. Gyamfi, J. M. Ndambuki, and R. W. Salim, "Simulation of sediment yield in a semi-arid River Basin under changing land use: an integrated approach of hydrologic modelling and principal component analysis," Sustainability, vol. 8, no. 11,2016. doi: 10.3390/su8111133.

[39] S. Yue, T. B. Ouarda, B. Bobée, P. Legendre, and P. Bruneau, "The Gumbel mixed model for flood frequency analysis," Journal of Hydrology, vol. 228, pp.88-100, 1999. doi:10.1016/S00221694(99)00168-7.

[40] J. T. Shiau, "Return period of bivariate distributed extreme hydrological events," Stochastic Environmental Research and Risk Assessment, vol. 17, pp.42-57, 2003. doi:10.1007/s00477-003-0125-9.

[41] S. Naz, M. J. Iqbal, S. M. Akhter, and I. Hussain, "The Gumbel mixed model for food frequency analysis of Tarbela," The Nucleus, 53(3), pp. $171-179,2016$ 